\title{
Levantamento dos problemas do dia a dia de um grupo \\ de amputados e dos dispositivos de auxílio que utilizam
}

\section{Survey of daily activity problems of a group of amputees and the aid devices they use}

\author{
Rubiani Ferracin Biffi', Alberto Luiz Aramaki², Fabiana Caetano Martins \\ Silva e Dutra ${ }^{3}$, Ivania Garavello ${ }^{4}$, Alessandra Cavalcanti ${ }^{5}$
}

http://dx.doi.org/10.11606/issn.2238-6149.v28i1p46-53

Biffi RF, Aramaki AL, Silva e Dutra FCM, Garavello I, Cavalcanti A. Levantamento dos problemas do dia a dia de um grupo de amputados e dos dispositivos de auxílio que utilizam. Rev Ter Ocup Univ São Paulo. 2018 jan.-abr.;28(1):46-53.

RESUMO: A amputação conduz a uma mudança no estilo de vida, limitando o envolvimento em atividades, restringindo a participação social e gerando dificuldades no desempenho funcional; implicando em perda de autonomia e independência para a mobilidade. A inclusão de tecnologia assistiva pode apoiar o envolvimento em atividades e favorecer a manutenção do desempenho ocupacional, oportunizando interação e envolvimento ativo da pessoa amputada em seu contexto. O presente estudo objetiva investigar quais os problemas que um grupo de amputados de membro inferior apresenta no seu cotidiano, e qual tecnologia assistiva eles possuem. Buscou-se também analisar se essa tecnologia auxilia no desempenho funcional dos membros inferiores dos indivíduos participantes da pesquisa. É um estudo de abordagem quantitativa, do tipo observacional transversal. Participaram do estudo 14 sujeitos que apontaram dificuldades de desempenho no cuidado pessoal, na mobilidade pessoal e na independência fora de casa; e relataram ter alguns recursos de tecnologia assistiva (cadeira de rodas, banco ou cadeira de banho, tapete de borracha). Concluiu-se que, na percepção dos usuários, os equipamentos de tecnologia assistiva em uso não suprem as demandas de atividades que desejam ou necessitam se envolver no dia a dia, não auxiliando no desempenho ocupacional.

DESCRITORES: Equipamentos de autoajuda; Amputação/ reabilitação; Extremidade inferior; Reabilitação.
Biffi RF, Aramaki AL, Silva e Dutra FCM, Garavello I, Cavalcanti A. Survey of daily activity problems of a group of amputees and the aid devices they use. Rev Ter Ocup Univ São Paulo. 2018 Jan.-Apr.;28(1):46-53.

\begin{abstract}
Amputation leads to a change in lifestyle, limiting involvement in activities, restricting social participation, and creating difficulties in functional performance; resulting in loss of autonomy and independence for mobility. The inclusion of assistive technology can support the involvement in activities and promote the maintenance of occupational performance, providing opportunities for interaction and active involvement of the amputee in context. This current study aims to investigate what problems an amputee group of lower limbs features in their day to day, and what assistive technology they have, analyzing whether they assist in functional performance. It is a study of quantitative approach, the cross observational type. The study included 14 subjects who showed day problems related to daily performance difficulties in personal care, personal mobility and independence outside home; and reported the presence of some assistive technology resources (wheelchair, bath seat or chair, rubber mat). It was concluded that the assistive technology equipment in use, in the perception of the users, do not meet the demands of involvement in activities that wish or need to engage in day to day, not helping in their occupational performance.
\end{abstract}

KEYWORDS: Self-help devices; Amputation/rehabilitation; Lower extremity; Rehabilitation.

Artigo decorrente do Trabalho de Conclusão de Curso (TCC) do Curso de Terapia Ocupacional da Universidade Federal do Triângulo Mineiro (UFTM) - aprovado pelo Comitê de Ética em Pesquisa com Seres Humanos da UFTM de acordo com o CAAE 39420414.7.0000.5154.

1. Terapeuta ocupacional graduada pela Universidade Federal do Triângulo Mineiro - UFTM. Estudante do Laboratório Integrado de Tecnologia Assistiva (LITA/UFTM).Email: rubiani_biffi@hotmail.com

2. Terapeuta ocupacional. Pós-Graduando do Programa de Pós-Graduação em Atenção à Saúde - UFTM. Estudante do Núcleo de Estudos e Pesquisas em Trabalho, Participação Social e Saúde (NETRAS). Estudante do Laboratório Integrado de Tecnologia Assistiva (LITA/ UFTM). Email: alberto_koston@hotmail.com

3. Professora Adjunta do Departamento de Terapia Ocupacional da Universidade Federal do Triângulo Mineiro - UFTM. Docente do Programa de Pós-Graduação em Atenção à Saúde - UFTM. Líder do Núcleo de Estudos e Pesquisas em Trabalho, Participação Social e Saúde (NETRAS). Email: fabiana.dutra@uftm.edu.br

4. Professora Adjunta do Departamento de Fisioterapia Aplicada. Pesquisadora do Laboratório Integrado de Tecnologia Assistiva. Curso de Fisioterapia. Universidade Federal do Triângulo Mineiro - UFTM. Email: garavelloi13@gmail.com

5. Professora Adjunta do Departamento de Terapia Ocupacional. Líder do Laboratório Integrado de Tecnologia Assistiva. Curso de Terapia Ocupacional. Universidade Federal do Triângulo Mineiro. Email: alessandra.cavalcanti@uftm.edu.br

Endereço para correspondência: A/C Alessandra Cavalcanti. Universidade Federal do Triângulo Mineiro. Unidade Centro Educacional (CE). Departamento de Terapia Ocupacional. Av. Getúlio Guaritá, nº 159, Térreo, Sala T21. Uberaba, MG. CEP: $38025-440$. 
Biffi RF, et al. Levantamento dos problemas do dia a dia de um grupo. Rev Ter Ocup Univ São Paulo. 2017 jan./abr.;28(1):46-53.

\section{INTRODUÇÃO}

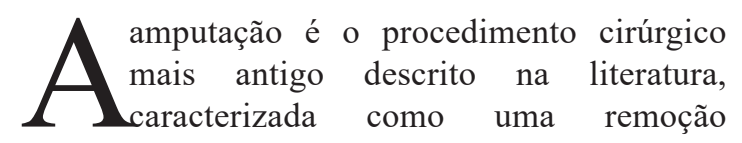
cirúrgica ou traumática (total ou parcial), ou um método reconstrutivo para segmento ou parte do corpo com função limitada ou sem função ${ }^{1}$. Inclui a retirada do tecido necrosado ou adoecido para alívio da dor, visando reparo tecidual e preparação de um coto para colocação de prótese com intuito de auxiliar na função do membro amputado ${ }^{4}$. Além das alterações vasculares principalmente advindas de diabetes, doenças vasculares periféricas e malignidades -, serem apontadas como causas frequentes de amputação ${ }^{2}$, nos últimos anos, devido ao aumento dos acidentes de trânsito, os traumas também têm sido apontados ${ }^{3}$.

Os membros inferiores, frequentemente, possuem maiores chances de serem submetidos ao procedimento de amputação em comparação aos membros superiores ${ }^{5}$. No Brasil, estima-se que as amputações de membro inferior correspondam a $85 \%$ do total. Segundo o Ministério da Saúde, 94\% das amputações realizadas até 2011 pelo Sistema Único de Saúde, foram de membro inferior ${ }^{6}$.

A amputação conduz a uma mudança no estilo de vida, alterando a capacidade do sistema músculo esquelético, limitando o envolvimento em atividades, restringindo a participação social e gerando dificuldades no desempenho funcional. É necessário adaptar-se a esta nova condição incapacitante que provoca alterações sociais, econômicas e familiares ${ }^{7}$. A incapacidade funcional relacionada à amputação de membros inferiores implica em perda de autonomia e independência para a mobilidade. Há uma redução na capacidade do amputado para realizar suas ocupações, isto é, as diferentes atividades cotidianas que ele necessita ou deseja se envolver ${ }^{7,8}$.

A incapacidade das pessoas amputadas de membro inferior pode estar relacionada para além da mobilidade, acometendo sua produtividade, prejudicando o desempenho em tarefas, como por exemplo, as de casa, do lazer ou do trabalho. A inclusão de tecnologia assistiva pode apoiar o envolvimento em atividades e favorecer a manutenção do desempenho ocupacional, oportunizando interação e envolvimento ativo da pessoa amputada em seu contexto".

Assim, o objetivo principal deste estudo foi investigar quais os problemas que um grupo de amputados de membro inferior apresenta no seu dia a dia, e qual tecnologia assistiva eles possuem. Também se buscou analisar se, considerando a percepção dos usuários participantes da pesquisa, tal tecnologia contribui no desempenho ocupacional.

\section{PROCEDIMENTOS METODOLÓGICOS}

Este trabalho é um estudo de abordagem quantitativa, do tipo observacional transversa ${ }^{10}$, aprovado pelo Comitê de Ética em Pesquisa da Universidade Federal do Triângulo Mineiro por meio do CAAE 39420414.7.0000.5154.

Os dados foram coletados entre os meses de fevereiro e junho de 2016 na unidade de reabilitação ambulatorial do complexo do Hospital de Clínicas da Universidade por uma equipe de três entrevistadores, pesquisadores do Laboratório Integrado de Tecnologia Assistiva da Universidade Federal do Triângulo Mineiro. Tal equipe foi treinada para a aplicação dos instrumentos e forma de abordagem junto aos participantes considerando o respeito às questões éticas.

Essa unidade de reabilitação ambulatorial é referência para atenção de média e alta complexidade em reabilitação física e por meio de uma parceria entre a Universidade e a Secretaria de Saúde do Município, realiza concessão de órteses, próteses e meios auxiliares de locomoção ambulatoriais pelo Sistema Único de Saúde (SUS) para 19 municípios que compõem a macrorregião do Triângulo Sul de Minas Gerais, incluindo a cidade de Uberaba.

Os instrumentos de coleta de dados foram divididos em quatro seções: uma primeira de caracterização dos participantes contendo gênero, idade, estado civil, causa da amputação, nível da amputação, tempo de amputação, tempo transcorrido até a triagem e até o início da reabilitação na unidade de reabilitação ambulatorial; uma segunda sessão era relacionada à classificação da classe econômica de acordo com o "Critério de Classificação Econômica Brasil - CCEB" da Associação Brasileira de Empresas de Pesquisa ${ }^{11}$; outra sessão mensurava o desempenho ocupacional dos participantes, sendo utilizada a Medida Canadense de Desempenho Ocupacional (COPM) ${ }^{12}$; e a quarta parte investigava o uso de tecnologia assistiva por meio de um formulário com respostas binárias (sim/não) proposto por Grandim e Cavalcanti ${ }^{13}$.

As questões relacionadas à classe econômica explicitam a renda média familiar dos participantes por classes, sendo: A ( $\$$ 20.272,56), B1 ( $\$$ \$ 8.695,88), B2 (R\$ 4.427,36), C1 (R\$2.409,01), C2 (R\$ 1.446,24), D-E (R\$ 639,78). 
Biffi RF, et al. Levantamento dos problemas do dia a dia de um grupo. Rev Ter Ocup Univ São Paulo. 2017 jan./abr.;28(1):46-53.

A Medida Canadense de Desempenho Ocupacional é um formulário que identifica atividades que são difíceis de realizar de forma satisfatória nas áreas de autocuidado, produtividade e lazer, classificando a importância de cada atividade para o entrevistado em uma escala de 1 a 10. As cinco atividades de maior importância apontadas como problemas são pontuados novamente pelo participante no que diz respeito ao seu desempenho e a sua satisfação também em uma escala de 1 a $10^{12}$.

As questões fechadas sobre uso de tecnologia assistiva agregaram informação sobre a presença/ ausência de dispositivos de assistência nas atividades de 'banho/tomar banho', 'alimentação', 'vestuário', 'uso do vaso sanitário', 'cuidado com equipamentos pessoais', 'mobilidade','higiene pessoal' e 'autocuidado'.

Nesta pesquisa, destaca-se ainda que a amostra foi por conveniência, sendo incluídas pessoas com amputação de membro inferior (transtibial ou transfemural), de ambos os gêneros,com idade acima de 18 anos e que estão em atenção na unidade de reabilitação, no período de coleta de dados descrito anteriormente. Somente participaram da pesquisa indivíduos que, após convite e esclarecimentos, assinaram o Termo de Consentimento Livre e Esclarecido. Não foram incluídas pessoas amputadas de membro inferior que estavam em atendimento por comorbidades associadas.

Os resultados coletados foram submetidos à análise descritiva por meio de frequência absoluta e relativa para as variáveis qualitativas (nível econômico, estado civil, gênero, diagnóstico clínico, escolaridade e tecnologia assistiva) e médias e desvio padrão para as variáveis quantitativas (idade, período da amputação, período da triagem, período de início da reabilitação, desempenho e satisfação). Todas as análises foram realizadas no software Statistical Package for Social Sciences (SPSS, Chicago, IL, USA) versão 20.

\section{RESULTADOS}

No período descrito de coleta de dados, foram identificadas 19 pessoas com diagnóstico de amputação do membro inferior em atendimento na unidade ambulatorial de reabilitação da Universidade. Dessas, apenas uma não aceitou participar do estudo e quatro foram excluídas por não estarem presentes nos atendimentos no período de coleta, totalizando uma amostra com 14 participantes.

A Tabela 1 apresenta as características sociodemográficas. A idade média dos participantes foi de 63,28 anos $(\mathrm{DP}=10,08)$, sendo a maior idade 80 anos e a menor 46 anos, com predomínio do gênero masculino
$(85,7 \%)$. Em relação ao estado civil, a maioria dos participantes declarou-se casado $(78,6 \%)$. A renda média foi de $\mathrm{R} \$ 1.537,65$ ( $\mathrm{DP}=1.334,07)$, com predomínio da classe econômica nível D-E (50,0\%). Quanto ao grau de instrução, a maioria dos participantes tinha o fundamental I incompleto (analfabeto ou primário incompleto) (71,5\%).

Tabela 1 - Características sociodemográficas dos participantes $(\mathrm{n}=14)$

\begin{tabular}{lcc}
\hline Variável & n & \% \\
\hline Gênero & & \\
\hline Feminino & 02 & 14,3 \\
\hline Masculino & 12 & 85,7 \\
\hline
\end{tabular}

Faixa Etária

\begin{tabular}{lll}
\hline $40-49$ & 02 & 14,3 \\
\hline $50-59$ & 02 & 14,3 \\
\hline $60-69$ & 05 & 35,7 \\
\hline $70-79$ & 04 & 28,6 \\
\hline $80-89$ & 01 & 07,1 \\
\hline
\end{tabular}

Estado Civil

\begin{tabular}{lll}
\hline Casado & 11 & 78,6 \\
\hline Viúvo & 03 & 21,4 \\
\hline Divorciado & 00 & 00,0 \\
\hline Solteiro & 00 & 00,0 \\
\hline
\end{tabular}

Grau de Instrução

\begin{tabular}{lll}
\hline Analfabeto/Fundamental I Incompleto & 10 & 71,5 \\
\hline Fund. I Completo/Fund. II Incompleto & 03 & 21,4 \\
\hline Fund. II Compl./Médio Incompleto & 01 & 07,1 \\
\hline Médio Compl./Superior Incompleto & 00 & 00,0 \\
\hline Superior Completo & 00 & 00,0
\end{tabular}

Nível Econômico

\begin{tabular}{lll}
\hline $\mathrm{A}$ & 00 & 00,0 \\
\hline $\mathrm{B} 1$ & 00 & 00,0 \\
\hline $\mathrm{B} 2$ & 02 & 14,3 \\
\hline $\mathrm{C} 1$ & 01 & 07,1 \\
\hline $\mathrm{C} 2$ & 04 & 28,6 \\
\hline $\mathrm{D}-\mathrm{E}$ & 07 & 50,0 \\
\hline
\end{tabular}

Com relação às causas da amputação, os participantes em sua maioria declararam terem sido submetidos à amputação decorrente de complicações de diabetes. Houve um relato de amputação decorrente de acidente automobilístico, um de acidente de trabalho e um terceiro por negligência a ferida com prego. Com relação aos níveis de amputação, 78,6\% dos entrevistados são amputados transtibial. O período entre a amputação e a 
Biffi RF, et al. Levantamento dos problemas do dia a dia de um grupo. Rev Ter Ocup Univ São Paulo. 2017 jan./abr.;28(1):46-53.

triagem na unidade de reabilitação ambulatorial foi de 4,85 meses $(\mathrm{DP}=4,48)$. Após a triagem, o tempo transcorrido para o início dos atendimentos na reabilitação teve média de 3,07 meses ( $\mathrm{DP}=1,49)$ (Tabela 2).

Tabela 2 - Etiologia, nível da amputação e tempo $(n=14)$

\begin{tabular}{|c|c|c|}
\hline Variável & $n$ & $\%$ \\
\hline \multicolumn{3}{|l|}{ Etiologia } \\
\hline Acidente de trânsito & 01 & 07,1 \\
\hline Acidente de trabalho & 01 & 07,1 \\
\hline Diabetes & 08 & 57,3 \\
\hline Negligência & 01 & 07,1 \\
\hline Trombose & 03 & 21,4 \\
\hline \multicolumn{3}{|l|}{ Nível } \\
\hline Transtibial (unilateral) & 11 & 78,6 \\
\hline Transfemural (unilateral) & 02 & 14,3 \\
\hline Transtibial e Transfemural (bilateral) & 01 & 07,1 \\
\hline \multicolumn{3}{|l|}{ Tempo de Amputação } \\
\hline Até 6 meses & 01 & 07,1 \\
\hline 6-12 meses & 02 & 14,3 \\
\hline 13-18 meses & 01 & 07,1 \\
\hline $19-24$ meses & 00 & 00,0 \\
\hline Acima de 24 meses & 10 & 71,5 \\
\hline \multicolumn{3}{|c|}{ Tempo da amputação à triagem na unidade } \\
\hline Até 1 mês & 05 & 35,7 \\
\hline $2-5$ meses & 04 & 28,6 \\
\hline Acima 6 meses & 05 & 35,7 \\
\hline \multicolumn{3}{|c|}{ Tempo entre triagem e início da reabilitação } \\
\hline Até 1 mês & 02 & 14,3 \\
\hline $2-3$ meses & 06 & 42,9 \\
\hline $4-5$ meses & 05 & 35,7 \\
\hline Acima 6 meses & 01 & 07,1 \\
\hline
\end{tabular}

Os problemas cotidianos apontados pelo grupo de amputados de membro inferior foram relacionados a atividades de 'auto cuidado', sendo registradas dificuldades de desempenho no cuidado pessoal, na mobilidade pessoal e na independência fora de casa. O grupo de participantes deste estudo citou poucas atividades 'produtivas' e de 'lazer' como difíceis de serem realizadas (Tabela 3). Quando avaliado o desempenho ocupacional e a satisfação, observou-se uma média de 4,27 escores $(\mathrm{DP}=2,47)$ e 3,32 escores $(\mathrm{DP}=1,83)$, respectivamente, sendo 8,3 a maior pontuação para desempenho e 7,0 para satisfação. O menor escore foi de 1,0 ponto para ambas as categorias de análise.
Tabela 3 - Problemas do dia a dia relatados $(n=14)$

\begin{tabular}{|c|c|c|c|c|}
\hline Categoria & Variável & & $n$ & $\%$ \\
\hline \multirow{11}{*}{ 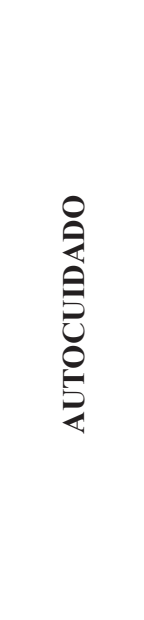 } & \multirow{4}{*}{$\begin{array}{l}\text { Cuidado } \\
\text { pessoal }\end{array}$} & Vestir membro inferior & 06 & 42,9 \\
\hline & & Banho & 04 & 28,6 \\
\hline & & Preparar refeições & 01 & 07,1 \\
\hline & & Higiene pessoal & 01 & 07,1 \\
\hline & \multirow{3}{*}{$\begin{array}{l}\text { Mobilidade } \\
\text { Pessoal }\end{array}$} & Transferência & 03 & 21,4 \\
\hline & & $\begin{array}{l}\text { Mobilidade dentro de } \\
\text { casa }\end{array}$ & 09 & 64,3 \\
\hline & & $\begin{array}{l}\text { Mobilidade fora de } \\
\text { casa }\end{array}$ & 11 & 78,6 \\
\hline & \multirow{4}{*}{$\begin{array}{l}\text { Independência } \\
\text { fora de casa }\end{array}$} & Transporte & 07 & 50,0 \\
\hline & & Compra & 04 & 28,6 \\
\hline & & Finanças & 02 & 14,3 \\
\hline & & Dirigir & 02 & 14,3 \\
\hline \multirow{4}{*}{ 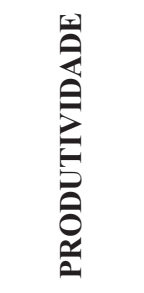 } & Trabalho & Trabalho & 00 & 00,0 \\
\hline & \multirow{3}{*}{$\begin{array}{l}\text { Tarefas } \\
\text { domésticas }\end{array}$} & Lavar roupa & 02 & 14,3 \\
\hline & & Limpar casa & 01 & 07,1 \\
\hline & & Cuidar do quintal & 01 & 07,1 \\
\hline \multirow{5}{*}{ 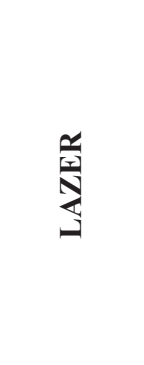 } & $\begin{array}{l}\text { Recreação } \\
\text { tranquila }\end{array}$ & Lavar carro & 01 & 07,1 \\
\hline & $\begin{array}{l}\text { Recreação } \\
\text { ativa }\end{array}$ & Viajar & 02 & 14,3 \\
\hline & \multirow[t]{3}{*}{ Socialização } & Ir à igreja & 01 & 07,1 \\
\hline & & Visitar amigos & 01 & 07,1 \\
\hline & & Ir a bar & 01 & 07,1 \\
\hline
\end{tabular}

Em relação à presença/ausência de tecnologia assistiva, foi observado que no item 'banho, tomar banho' a maioria possui cadeira de banho e tapete de borracha como dispositivos de assistência. No 'vestuário', apenas dois participantes relataram uso de prótese; em 'equipamentos pessoais' o uso de óculos foi descrito por $50 \%$ dos participantes. Já no item 'uso do vaso sanitário', apenas $21,4 \%$ relatou possuir barras de segurança lateral como equipamento de assistência para a realização desta atividade. E quanto à 'mobilidade', constatou-se que a maioria dos participantes $(57,1 \%)$ possuía mais de um equipamento de assistência (por 
Biffi RF, et al. Levantamento dos problemas do dia a dia de um grupo. Rev Ter Ocup Univ São Paulo. 2017 jan./abr.;28(1):46-53.

exemplo, cadeira de rodas e andador), sendo relatado por $85,7 \%$ o uso de cadeira de rodas para mobilidade (Tabela 4).

Tabela 4 - Tecnologia assistiva existente em atividades diárias $(n=14)$

\begin{tabular}{lcc}
\hline BANHO, TOMAR BANHO & $\boldsymbol{n}$ & $\boldsymbol{\%}$ \\
\hline Banco ou cadeira para banho & 12 & 85,7 \\
Tapete de borracha com ventosa & 10 & 71,5 \\
Esponja ou escova de cabo longo & 04 & 28,6 \\
Barra de segurança e apoio & 03 & 21,4 \\
\hline VESTIR-SE & $\boldsymbol{n}$ & $\boldsymbol{\%}$ \\
\hline Prótese & 02 & 14,3 \\
\hline CUIDADO COM EQUIPAMENTOS & $\boldsymbol{n}$ & $\boldsymbol{\%}$ \\
PESSOAIS & 01 & 07,1 \\
\hline Aparelho Auditivo & 07 & 50,0 \\
Óculos & $\boldsymbol{n}$ & $\boldsymbol{\%}$ \\
\hline USO DO VASO SANITARIO & 03 & 21,4 \\
\hline Barra de segurança e apoio & $\boldsymbol{n}$ & $\boldsymbol{\%}$ \\
\hline MOBILIDADE & 05 & 35,7 \\
\hline Andador & 06 & 42,9 \\
Muleta & 12 & 85,7 \\
Cadeira de rodas &
\end{tabular}

(*) Em 'Alimentação' e 'Higiene Pessoal' não foram identificados tecnologia assistiva.

\section{DISCUSSÃO}

A amputação de membro inferior resulta em mudanças expressivas na autonomia, no envolvimento em tarefas e atividades e comumente leva o amputado à restrições de participação em situações que lhe são comuns, desejadas ou necessárias ao longo de um dia. Ao considerar os resultados encontrados, a maioria dos participantes eram homens, casados, com baixo grau de instrução e com renda média mensal menor que um salário mínimo. A maioria tem idade acima de 60 anos e declarou não estar trabalhando. Semelhante aos achados deste estudo, Ganz ${ }^{14}$ registra que $75 \%$ dos amputados de membro inferior são do gênero masculino e o Ministério da Saúde declara que a frequência é maior em idosos ${ }^{6}$.

A maior parte dos entrevistados é amputado em um único membro, nível transtibial (78,6\%). A amputação com esta característica é a mais realizada entre as amputações de membro inferior ${ }^{1,15,16}$. Dados apontados em outro estudo $^{17}$ relatam que nos Estados Unidos cerca de 1,3 milhões de pessoas são amputadas na extremidade inferior, e, dentre elas, $28 \%$ foram amputadas no nível transtibial. Esse nível compreende toda amputação realizada entre as articulações do tornozelo/pé e joelho preservando a articulação do joelho, o que favorece a diminuição do gasto energético para movimentação do membro inferior durante a marcha, além de permitir um coto que facilita a colocação e remoção da prótese ${ }^{18}$.

No grupo de amputados, 57,3\% das etiologias relatadas pelos participantes como causa da amputação foi diabetes e $21,4 \%$ trombose, o que apóia as considerações de Luccia e Silva ${ }^{19}$ sobre a diabetes e/ou doença vascular periférica serem as causas de $80 \%$ das amputações de membros inferiores no Brasil. As exposições de Gamba et al. ${ }^{20}$ também confirmam ser cada vez mais frequente no país e no mundo as amputações de membros inferiores devido a complicações decorrentes da diabetes.

Grande parte dos amputados relatou dificuldades nas atividades de mobilidade (64,3\% dentro de casa e $78,6 \%$ fora de casa), de vestuário (vestir/despir calça, bermuda, por exemplo) e para usar transporte (50\% público e 14,3\% particular). Esses achados são compatíveis com a observação de que a maioria é amputado transtibial $(78,6 \%)$ e faz uso da cadeira de rodas $(85,7 \%)$ como o equipamento de assistência para o deslocamento.

Para o Ministério da Saúde $^{6}$, as transferências e os deslocamentos, assim como o treino de marcha, são atividades pertencentes à fase pós-cirúrgica imediata no tratamento da pessoa amputada. Portanto, o programa de reabilitação na fase inicial de atenção deveria estar focado no condicionamento físico e no treino de marcha com progressão nos equipamentos (barras paralelas, andador, muletas axilar ou canadense) de forma a minimizar as queixas observadas. Entretanto, outros autores ${ }^{21}$ esclarecem que mesmo com a atenção preconizada, pessoas amputadas de membro inferior apresentarão limitação na mobilidade durante meses ou anos após a retirada da extremidade.

Além disso, dos entrevistados, apenas dois possuem prótese e estão na fase protética, porém relataram dificuldades na mobilidade dentro e fora de casa e apresentaram baixo desempenho e satisfação com as atividades realizadas diariamente.

O uso do equipamento de tecnologia assistiva do tipo cadeira de rodas pela maioria dos participantes $(85,7 \%)$ corrobora as orientações do Ministério da Saúde ${ }^{6}$, sobre este ser o equipamento comum aos amputados transtibial na fase inicial de reabilitação. No entanto, para a amostra desta pesquisa não era esperado encontrar a cadeira de rodas como o dispositivo de tecnologia assistiva de maior representação, pois 92,8\% dos participantes não se encontram na fase inicial do processo de reabilitação. O que se observou foi um alto percentual de participantes em estágio avançado da reabilitação sem uso de prótese $(85,7 \%)$. No município de Uberaba - MG, a acessibilidade 
Biffi RF, et al. Levantamento dos problemas do dia a dia de um grupo. Rev Ter Ocup Univ São Paulo. 2017 jan./abr.;28(1):46-53.

é restrita devido a geografia do terreno, as características de construção urbana, e a ausência de políticas de mobilidade e de acesso a diferentes espaços, de forma a oportunizar a inclusão e independência de pessoas com deficiência. Assim, a dificuldade de mobilidade relatada está relacionada também a fatores ambientais e a diferentes características associadas ao seu contexto.

Moro et al. ${ }^{22}$, investigando amputados de membro inferior, identificaram que mais de $60 \%$ dos entrevistados faziam uso de prótese, o que contraria o cenário identificado neste estudo. É importante ressaltar que a unidade ambulatorial de reabilitação além de ser referência para outras cidades que compõem a região, também é o local de dispensação de prótese. Essa diversidade nos resultados conduz à reflexão sobre a linha de cuidado e o processo de reabilitação oferecido para a pessoa amputada, assim como sobre os procedimentos relacionados à concessão de equipamentos a estes usuários.

Por outro lado, Marshall e Stansby ${ }^{23}$ descrevem que o exame minucioso do nível de amputação deve levar em consideração as possibilidades de uma reabilitação voltada à independência e autonomia da pessoa. Tais autores destacam que ao usar uma prótese abaixo do joelho, a pessoa tem um dispêndio de $25 \%$ a $40 \%$ a mais de energia para desempenhar marcha e, para uma prótese acima do joelho, este valor sobe para $65 \%$ a $100 \%{ }^{23}$. Assim, os autores pontuam que o uso de cadeira de rodas exige um gasto de energia de apenas $8 \%$ a mais do que aquele desempenhado na locomoção de uma pessoa não amputada ${ }^{23}$. No entanto, frente aos escores de desempenho e satisfação apontados pelos participantes, é importante analisar se a diminuição de gasto energético com o uso de cadeira de rodas justifica as limitações e restrições de mobilidade dentro de casa e mobilidade na rua que este equipamento impõe.

Quando se associa o nível da amputação, a idade e os problemas em atividades/tarefas diárias relatadas pelos entrevistados se verifica pouca relação entre os resultados observados. Parte dos participantes está em idade ativa, poucos usuários citaram dificuldades em atividades produtivas e de lazer e dois entrevistados, apesar de fazerem uso de prótese, apresentam as mesmas restrições relatadas pelos participantes usuários de cadeiras de rodas. A perda da funcionalidade em pacientes amputados deve então ser associada com cautela à condição de saúde propriamente dita. Embora a maior parte das pessoas com amputações apresente múltiplas limitações funcionais, a amputação em si não implica em dependência. Outros fatores irão determinar o que uma pessoa amputada pode fazer, como os contextos nos quais vivem e suas interações neles. A diversidade no desempenho funcional e nas capacidades de pessoas com amputações não é aleatória e o contexto pode fornecer uma gama de recursos ou barreiras que influenciarão na funcionalidade destas pessoas.

Neste sentido, Parker et al..$^{24}$ explicitam que existe um limite tênue entre o que uma pessoa consegue realizar (sua capacidade) e o que ela realmente realiza (seu desempenho). Os participantes têm condições de se envolverem em atividades que desejam ou que necessitam realizá-las de forma independente, porém não conseguem ultrapassar o limiar entre a capacidade e o desempenho provavelmente devido à assistência dispensada, à ausência de tecnologia assistiva adequada e/ou às condições socioeconômicas e emocionais. Sobre este aspecto, destacam-se as considerações de Rathore et al. ${ }^{1}$ que, ao contextualizarem as amputações no membro inferior, contrapõem os procedimentos e resultados do século passado aos avanços das técnicas cirúrgicas, das sistematizações dos cuidados pós-operatórios, do acesso à reabilitação multidisciplinar e à qualidade de próteses, que conduziriam a bons resultados funcionais e maior inserção na comunidade.

Dessa forma, há uma necessidade de reavaliar e redirecionar o processo da reabilitação que é dispensada na unidade que compôs este estudo, de forma a entender a composição da equipe multiprofissional que o acompanha e as ações que são pactuadas no programa de reabilitação, para se apontar como os equipamentos de tecnologia assistiva são inseridos no cotidiano desses usuários do Sistema Único de Saúde. Da mesma forma, é importante implementar um processo de respostas às complexas questões que envolvem a atenção à saúde das pessoas com deficiência e delinear um processo de reabilitação mais voltado para as demandas funcionais do usuário. $\mathrm{O}$ escore de desempenho e da satisfação legitima este cenário, uma vez que as médias da COPM mostraram valores de moderado a baixo. Destaca-se que estes valores geralmente são pontuações atribuídas por usuários do serviço que iniciam um processo de reabilitação, com perdas funcionais típicas de lesões recentes. No entanto, apesar da maioria dos participantes já estar em reabilitação há mais de um ano $(71,4 \%)$, os mesmos relataram não conseguirem realizar as atividades cotidianas que desejam e não estão satisfeitos com a forma que desempenham suas ocupações.

Outras evidências também apontam para a importância de se considerar os fatores contextuais no processo de reabilitação de pessoas amputadas de membro inferior. Em um estudo ${ }^{25}$ comparativo entre amputados de membro inferior dos Estados Unidos e da Tanzânia, aqueles com baixo suporte social (incluindo neste item a renda familiar) e que foram identificados precocemente pela equipe, tiveram ações para uma assistência direcionada 
Biffi RF, et al. Levantamento dos problemas do dia a dia de um grupo. Rev Ter Ocup Univ São Paulo. 2017 jan./abr.;28(1):46-53.

para integração social e apresentaram resultados funcionais e qualidade de vida mais significativos após a amputação. Assim, no processo de reabilitação, ações voltadas para participação em situações sociais apoiariam resultados positivos para mobilidade funcional e na comunidade, no mínimo para pequenas distâncias ${ }^{21}$.

\section{CONCLUSÃO}

Os resultados desta pesquisa apontam que grande parte dos amputados de membro inferior participantes possui problemas de desempenho nas atividades de mobilidade dentro de casa e fora de casa, de vestuário (vestir/despir calça, bermuda, por exemplo) e para usar transporte público e particular. A maioria utiliza como recurso de tecnologia assistiva a cadeira de banho e o tapete de borracha, além de mais de um equipamento de assistência para a tarefa de mobilidade (por exemplo, cadeira de rodas e andador). A cadeira de rodas foi o dispositivo de assistência mais relatado, ao invés da prótese de membro inferior.

Os participantes desta pesquisa também relataram utilizarem recursos de tecnologia assistiva para atividades de cuidado pessoal, mobilidade e independência fora de casa, porém os equipamentos em uso (por exemplo, cadeira de rodas para mobilidade ao invés de prótese) não suprem as demandas de envolvimento em atividades que desejam (por exemplo, mobilidade fora de casa) ou necessitam (por exemplo, uso de transporte público) se envolver no dia a dia, resultando em baixos escores para desempenho e satisfação ocupacional.

Por fim, cabe mencionar que ainda são necessários outros estudos que visem compreender o processo de concessão de prótese para membro inferior, levando em consideração o tempo transcorrido entre o pedido e a chegada do equipamento. E ampliar as ações de melhoria do serviço prestado de forma a auxiliar as pessoas amputadas a manterem os níveis de autonomia e independência.

\section{REFERÊNCIAS}

1. Rathore FA, Ayaz SB, Mansoor SN, Qureshi AR, Fahim M. Demographics of lower limb amputations in the Pakistan Military: a single center, three-year prospective survey. Cureus. 2016;8(4):e566. doi: 10.7759/cureus.566.

2. Gabarra LM. Estados emocionais, formas de enfrentamento, rede de apoio e adaptação psicossocial em pacientes amputados [Dissertação]. Florianópolis: Universidade Federal de Santa Catarina; 2010.

3. Reis G, Júnior AJC, Campos RS. Perfil epidemiológico de amputados de membros superiores e inferiores atendidos em um centro de referência. Rev Eletr Saúde Ciên. 2012;2(2):52-62. Disponível em: http://www.rescceafi.com. $\mathrm{br} / \mathrm{vol} 2 / \mathrm{n} 2 /$ Gleycykely-dos-Reis-52-62.pdf.

4. Santos LF, Fritzen PG, Gonçalves BR, Melo SA, Silva VF. Perfil das amputações de membros inferiores de pacientes cadastrados na associação de deficientes físicos de Apucarana Saúde Pesqui. 2010;3(1):59-64.

5. Santos FS. Avaliação e análise cinemática da marcha em pacientes amputados transfemorais pré e pós-aplicação de um protocolo específico de reabilitação: um estudo de caso [Dissertação]. Ribeirão Preto: Programa de aprimoramento Profissional; 2013.

6. Brasil. Ministério da Saúde. Secretaria de Atenção à Saúde. Departamento de Ações Programáticas Estratégicas.
Diretrizes de atenção à pessoa amputada. 2a ed. Brasília: Ministério da Saúde; 2014.

7. Silveira AC, Bahia LAC, Peixoto LM, Diaz MCN, Izukawa NM. Reabilitação nos pacientes submetidos a amputações maiores no Instituto Dante Pazzanese de Cardiologia. São Paulo: IDPC; 2014.

8. The American Occupational Therapy Asssociation. Occupational Therapy practice: framework: domain \& process. 3a ed. Am J Occup Ther. 2014;68(Suppl 1):S1-48. doi: 10.11606/issn.2238-6149.v26iespp1-49

9. Cruz DMC, Rodrigues DS, Matsushima AM, Santos P, Figueiredo MO. O trabalho e a tecnologia assistiva na perspectiva de pessoas com deficiência física. Rev Ter Ocup Univ São Paulo. 2015;26(3):382-9. doi: 10.11606/issn. 2238-6149.v26i3p382-389.

10. Sampieri RH, Collado CF, Lucio PB. Metodologia de pesquisa. São Paulo: MacGrawHill; 2006.

11. Associação Brasileira de Empresas de Pesquisa. Critério Brasil [citado 04 fev 2016]. São Paulo; 2015. Disponível em: http://www.abep.org/criterio-brasil.

12. Law M, Magalhães LC, Magalhães LV, Cardoso AA. Medida canadense de desempenho ocupacional. Belo Horizonte: Editora UFMG; 2009. 
Biffi RF, et al. Levantamento dos problemas do dia a dia de um grupo. Rev Ter Ocup Univ São Paulo. 2017 jan./abr.;28(1):46-53.

13. Grandim LC, Cavalcanti A. Projeto de pesquisa recursos da tecnologia assistiva na atenção básica: formulário de coleta de dados. Uberaba: LITA/UFTM; 2012.

14. Ganz D. Tratado de reabilitação: princípios e prática. 3a ed. São Paulo: Ed. Manole; 2002.

15. Pires SR, Sandoval RA. Perfil de diabéticos amputados de membro inferior atendido no serviço de fisioterapia do centro de reabilitação e readaptação Dr. Henrique Santillo Crer. Trances. 2010;2(4):213-24. Disponível em: http:// www.trances.es/papers/TCS\%2002_4_1.pdf.

16. Singh BG, Pithawa AK, Ravindranath G. Study of disabled treated at artifical limb centre. Med J Armed Forces India. 2009;65:232-4. doi: 10.1016/S0377-1237(09)80010-5.

17. Highsmith JM, Kahle JT, Miro RM, Orendurff MS, Lewandowski AL, Orriola JJ, et al. Prosthetic interventions for people with transtibial amputation: systematic review and meta-analysis of high-quality prospective literature and systematic reviews. J Rehabil Res Dev. 2016;53(2):157-84. doi: 10.1682/JRRD.2015.03.0046.

18. Carvalho J. A. Amputações de membros inferiores: em busca da plena reabilitação. 2a ed. São Paulo: Manole; 2003.

19. Luccia N, Silva ES. Aspectos técnicos de amputações de membros inferiores. In: Pitta G. Angiologia e cirurgia vascular: guia ilustrado. Rio de Janeiro: Guanabara Koogan; 2003.
20. Gamba MA, Gotlieb SLD, Bergamaschi DP, Vianna AC. Amputações de extremidades inferiores por diabetes mellitus: estudo caso-controle. Rev Saude Publica. 2004;38(3):399-404. doi: 10.1590/S003489102004000300010 .

21. Amtmann D, Sara J, Morgan SJ, Kim J, Hafner, BJ. Health-related profiles of people with lower limb loss. Arch Phys Med Rehabil. 2015;96:1474-83. doi: 10.1016/j. apmr.2015.03.024.

22. Moro A, Assef MG., Araújo SW. Avaliação da qualidade de vida em pacientes submetidos à amputação de membros inferiores. Arq Catarin Med. 2012;41(1):41-6.

23. MarshallC, StansbyG.Amputationandrehabilitation. Surgery. 2010;28 (6):284-7. doi: 10.1016/j.mpsur.2010.01.017

24. Parker K, Kirby RL, Adderson J, Thompson K. Ambulation of people with lower-limb amputations: relationship between capacity abd performance measures. Arch Phys Med Rehabil. 2010;91:543-9. doi: 10.1016/j.apmr.2009.12.009.

25. Hawkins AT, Pallangyo AJ, Herman AM, Schaumeier MJ, Smith AD, Hevelone ND, et al. The effect of social integration on outcomes after major lower extremity amputation. J Vasc Surg. 2016;63(1):154-62. doi: 10.1016/j. jvs.2015.07.100. 